\title{
Permanent Magnet Synchronous Machine Non-Uniform Demagnetization Detection Using Zero-Sequence Magnetic Field Density
}

\author{
David Reigosa ${ }^{*}$, Daniel Fernández ${ }^{*}$, María Martínez ${ }^{*}$, Yonghyun Park ${ }^{* *}$, Sang Bin Lee ${ }^{* *}$ and Fernando Briz \\ "University of Oviedo. Dept. of Elect., Computer \& System Engineering, Gijón, 33204, Spain. \\ Korea University, Dept. of Elect. Engineering, Seoul, Korea. \\ diazdavid@uniovi.es, fernandezalodaniel@uniovi.es, martinezgmaria@uniovi.es, yonghyun.park@eecs.korea.ac.kr, \\ sangbinlee@korea.ac.kr, fernando@isa.uniovi.es
}

\begin{abstract}
Demagnetization in permanent magnet synchronous machines (PMSMs) results in a decrease in the motor average torque and could also increase the torque ripple and consequently vibration and acoustic noise, degrading the overall performance and reliability of the drive. Demagnetization detection can be therefore of tremendous importance. Use of hall-effect sensors has proven to be a viable option for demagnetization detection. Results reported using this method were based on the analysis of the flux density complex vector; the accuracy of this implementation strongly depends on the angle of the hall-effect sensor, being also sensitive to implementation issues such as offsets and gain variations of the sensors, PMs' temperature, manufacturing tolerances and stator current injection. This paper proposes the use of the zero-sequence component of the magnetic flux density for demagnetization detection. The use of this signal will improve the accuracy and reduce the sensitivity to implementation issues compared to the use of the flux complex vector. ${ }^{1}$
\end{abstract}

Keywords- Permanent magnet synchronous machines, nonuniform demagnetization, Hall-effect sensors.

\section{Introduction}

Design and control of PMSMs has been the focus of significant research efforts during the last three decades due to their good dynamic performance, power density and efficiency. PMs based on rare earths, e.g. Neodymium-IronBoron $(\mathrm{NdFeB})$, are the most common option [1]-[3]. Torque production capability of PMSMs depends on the magnetization state of the PMs [1]-[2]. A decrease of the PMs magnetization state, e.g. due to temperature increase [1],[4][6] or to the stator current [7]-[8], results in a decrease of the motor torque and efficiency. Demagnetization can occur locally (i.e. partial demagnetization within a PM or asymmetric magnetization among poles) or globally (i.e.

\footnotetext{
1 This work was supported in part by the Research, Technological Development and Innovation of the Spanish Ministry Economy and Competitiveness, under grant MINECO-17-ENE2016-80047-R and by the Economic Development Agency of the Principality of Asturias (IDEPA) \& ThyssenKrupp under grant SV-PA-17-RIS3-2.
}

uniform demagnetization of all PMs) [9]-[10]. Global demagnetization typically results in a decrease of the average torque; local demagnetization can also produce an increase of the torque ripple, motor vibration and acoustic noise. PM demagnetization detection in PMSMs is therefore of great importance.

Magnetization state can be measured by inserting a gauss meter in the machine air gap [10]-[11]; removing or drilling the end frame of the machine is required to insert the field sensor, field measurement being feasible only with the machine at standstill. Use of field sensors inserted between PMs and rotor lamination was proposed in [12]. Combined with a wireless transmission of the signal, this system provides real time measurement of the magnetization state and without interfering with the normal operation of the machine. Unfortunately this solution is expensive and compromises the robustness of the drive, being therefore unacceptable in most applications.

Alternatively to direct measurement, PM magnetization state can be estimated. Global [13]-[18] and local [18]-[26] demagnetization detection/estimation methods have been already proposed. Global demagnetization estimation can be performed using BEMF [13]-[14], signal injection [15]-[17] and hall-effect sensor [18] based methods. BEMF and signal injection methods estimate the magnetization state from stator terminal variables; BEMF requires the machine to be rotating, estimation at low speed or standstill not being possible. On the other hand, signal injection methods place concerns because of the potential adverse effects (additional noise, vibration and losses) due to the injected signal. Local demagnetization estimation methods include stator current analysis [19]-[20], zero-sequence voltage [21], BEMF [22], vibration analysis [23]-[24], change in the shaft trajectory [25], signal injection [26] and hall-effect sensors [18]. Stator current analysis, zero-sequence voltage, BEMF, vibration analysis and changes in the shaft trajectory methods require the machine to be rotating; signal injection methods require the machine to be at standstill, with the disadvantage of requiring the injection of an additional signal; as for the global demagnetization case.

Demagnetization detection using hall-effect sensors was proposed in [18]. Hall-effect sensors already present in many PMSM drives for magnet polarity and initial position 
detection [27]-[29] can be used for indirect measurement of the PM flux, enabling detection of partial and global magnetization at any speed and without interfering with the normal operation of the machine. The implementation proposed in [18] was based on the analysis of the magnetic flux density complex vector. A conclusion of that work was that measuring the flux in the radial direction is preferred over measurements in the tangential or axial directions in terms of reliability/sensitivity requirements. In addition, the method was demonstrated to be sensitive to several implementation issues, offsets in the sensors, unbalances in sensors' gains, manufacturing tolerances, stator current effects and magnets' temperature being the most relevant.

This paper proposes the use of the zero-sequence component of the magnetic flux density for demagnetization detection. It will be shown that the use of the zero-sequence component significantly reduces the sensitivity to several implementation issues, including sensors location, DC offsets and gain variations of the sensors, PMs' temperature or stator current injection.

The paper is organized as follows: Magnetic flux density measurement and zero-sequence component calculation in PMSMs is discussed in section II; zero-sequence magnetic flux density modeling of a partially demagnetized PM is described section III; experimental verification of non-uniform demagnetization detection using the zero-sequence magnetic flux density is discussed in section IV; discussion on implementation issues is included in section V; conclusions are finally presented in section VI.

\section{Magnetic flux density measurement and zero-sequence component calculation in PMSMs}

This section discusses the measurement and processing of the zero-sequence magnetic flux density component. Schematic design of the machine is shown in Fig. 1a and $1 \mathrm{~b}$. The machine is equipped with Neomax 42-SH PMs. Dimensions and ratings of the test machine are shown in Table I. Hall-effect sensors are attached to the end shield as shown in Fig. 1c. The end shield was modified to allow insertion/extraction of the PMs without the need of removing the rotor/end shield (rectangular window in Fig. 1d).

\begin{tabular}{|l|l|}
\hline \multicolumn{2}{|c|}{ Table I. Machine parameters } \\
\hline$P_{\text {RATED }}(\mathrm{kW})$ & 7.5 \\
\hline$I_{\text {RATED }}(\mathrm{A})$ & 14 \\
\hline$\omega_{\text {RATED }}(\mathrm{rpm})$ & 1800 \\
\hline Stator slots & 36 \\
\hline Poles & 6 \\
\hline Rotor radius $(\mathrm{mm})$ & 54.2 \\
\hline Magnets & $\mathrm{N} 42 \mathrm{SH}$ \\
\hline Magnet dimensions: width, height and length $(\mathrm{mm})$ & $42 \times 6 \times 10$ \\
\hline Magnet position from shaft center $(\mathrm{mm})$ & 44.6 \\
\hline Magnet position, $x y z(\mathrm{~mm})$ & $0,0,6$ \\
\hline Airgap length $(\mathrm{mm})$ & 0.8 \\
\hline Inner stator radius $(\mathrm{mm})$ & 55 \\
\hline Outer stator radius $(\mathrm{mm})$ & 88 \\
\hline
\end{tabular}

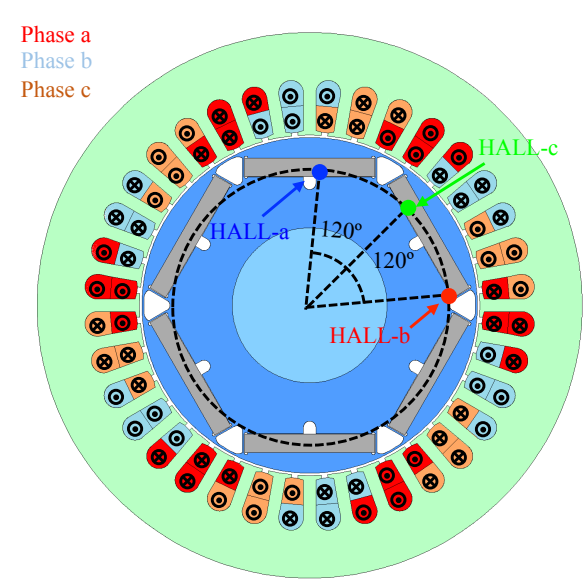

a)

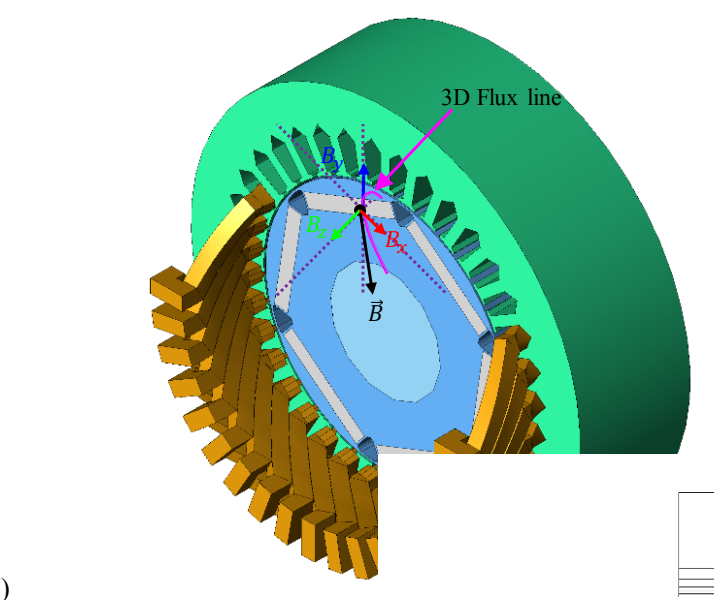

b)
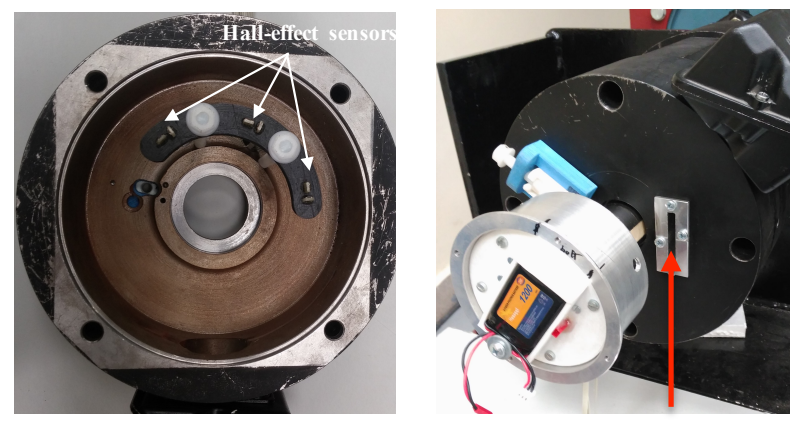

d)

window

e)

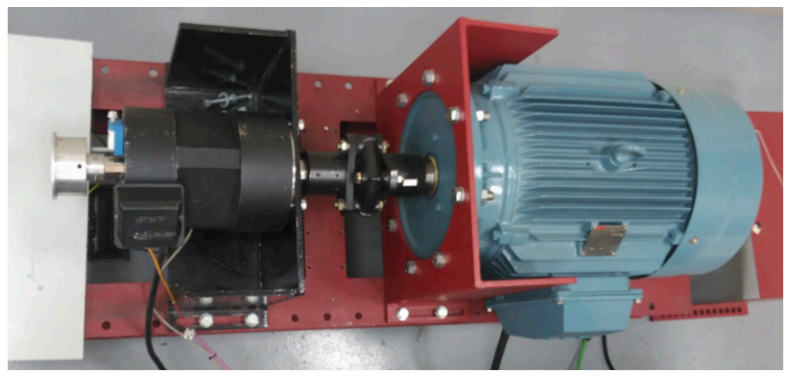

Fig. 1.- Machine desing and experimental setup. a) $2 \mathrm{D}$ and b) 3D schematic representation of the machine and sensors location; $x, y$ and $z$ correspond to the tangential, radial and axial directions respectively. c) Machine end shield with the hall-effect sensors, d) machine shield modification for easy PM replacement, and e) test bench. 


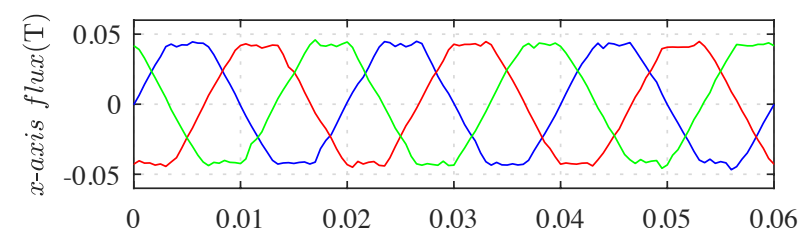

a)

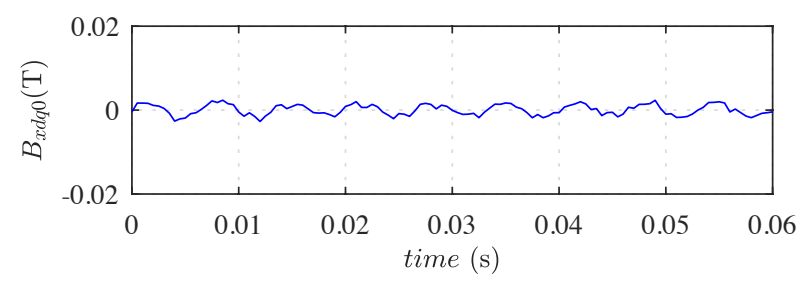

b)

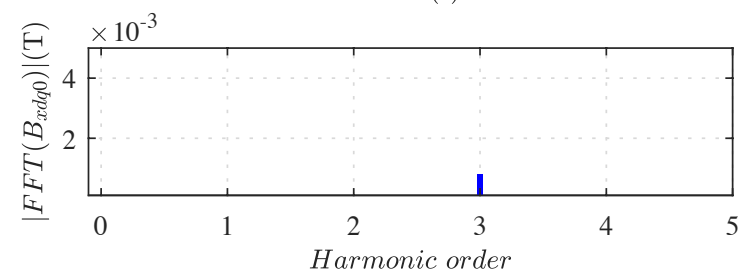

Fig. 2.- a) flux measurements in the $x$-axis direction by the three analog hall effect sensors ( $B_{x a}, B_{x b}$ and $\left.B_{x c}\right)$, b) resulting zero-sequence magnetic flux density, $B_{x d q 0}$,d) and FFT of $B_{x d q 0}$. $x=0 \mathrm{~mm}, y=0 \mathrm{~mm}$, $z=5 \mathrm{~mm}, \omega_{r}=1 \mathrm{pu}$ and $i_{d q}=0 \mathrm{pu}$.
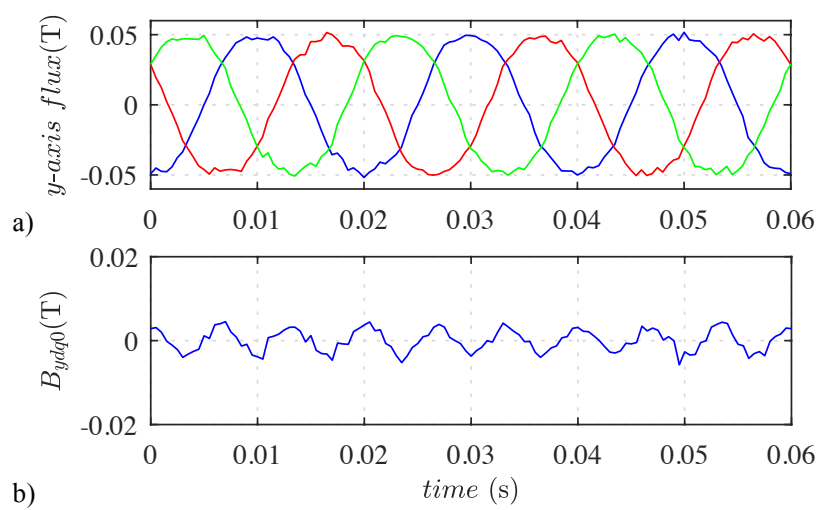

b)

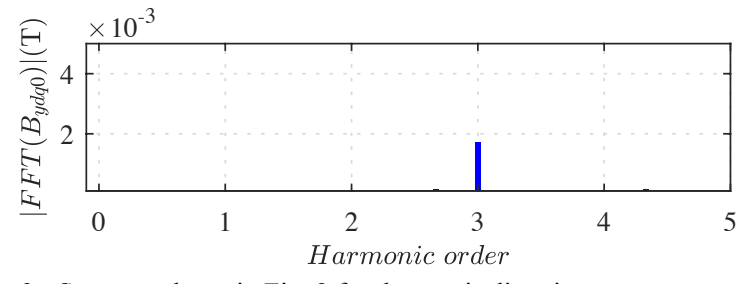

Fig. 3.- Same results as in Fig. 2 for the $y$-axis direction.

Previous to insertion in the rotor, PMs are magnetized/demagnetized using a pulse magnetizer [16]-[18]. Fig. 1d shows the test bench, which consists of the test machine and a load machine. Standard hall-effect sensors used in PMSMs drives for motion control [30] will be used in this work. Though practical implementation of the method would use standard 1D sensors, an arrangement of three sensors able to measure along $x, y$ and $z$ (radial, tangential and axial) directions has been developed to analyze the influence of measurement angle.

The zero-sequence magnetic flux density is defined by (1), where $m$ stands for $x, y$ or $z$-axis and $B_{m a}, B_{m b}$ and $B_{m c}$, are the magnetic flux densities measured by sensors $a, b$ and $c$ along the $m$ direction respectively.

$B_{m d q 0}=1 / 3\left(B_{m a}+B_{m b}+B_{m c}\right)$

An example of the measured magnetic flux density along the $x$-axis direction for one rotor revolution is shown in Fig. $2 \mathrm{a}$. Fig. $2 b$ shows the resulting zero-sequence magnetic flux density, $B_{x d q 0}$, while Fig. $2 \mathrm{c}$ shows the FFT of $B_{x d q 0}$. Since the measured zero sequence flux density must be a periodic function over a whole mechanical revolution, its frequency spectrum can be expressed as (2), where $\omega_{r}$ is the machine speed, and $B_{m d q 0_{-} n \omega r}^{s}$ and $\varphi_{n m}$ are the magnitude and phase of the " $n$ "th" harmonic component.

$B_{m d q 0}=\sum_{n} B_{m d q 0_{-} n \omega r} \sin \left(n \omega_{r} t+\varphi_{n m}\right)$

It is observed from Fig. 2c that the main harmonic component of $B_{x d q 0}$ is a third harmonic. Fig. 3 shows similar results as Fig. 2 but when the field is measured along $y$-axis direction; measurements in both axes are seen to behave very similar.

It is finally noted that, from the experimental results obtained in this work, the flux density in the $\mathrm{z}$-axis direction is significantly smaller compared to $x$ and $y$-axis for the specific case where the sensor is located at $x=y=0 \mathrm{~mm}$ (see Table I). Since this prevents reliable detection of demagnetization, measurements in the z-axis will not be therefore discussed further.

\section{Zero-sequence magnetic flux density modeling of a partially demagnetized PM}

It is useful for analysis purposes to develop models able to predict the effect of demagnetization of the flux measured by the sensors. Fig. 4a and Fig. 5a show the ideal flux measured by the three analog hall-effect sensors $\left(B_{m a}, B_{m b}\right.$ and $\left.B_{m c}\right)$ in the $x$ and $y$-axis directions respectively for the case of an ideal machine (i.e. purely sinusoidal waveforms). These ideal results agree with the experimental results shown in Fig. 2 and Fig. 3. Fig. $4 \mathrm{~b}$ and Fig. $5 \mathrm{~b}$ show the corresponding zero-sequence magnetic flux densities, $B_{m d q 0}(1)$, for the $x$ and $y$-axis directions respectively. In case of a demagnetized PMs, the flux measured by the hall-effect sensors will not be sinusoidal anymore but shaped by the demagnetized PMs. Such shaped flux measurements can be modeled as the product of the signals shown in Fig. $4 \mathrm{a}$ and Fig. $5 \mathrm{a}$ by a window function. Such window functions will be identical for sensors $a, b$ and $c$ but shifted by 120 electrical degrees. Windows functions of the type shown in Fig. $4 \mathrm{c}$ and Fig. $5 \mathrm{c}$ have been found to fit well with experimental measurements.

Fig. $4 \mathrm{~d}$ and Fig. $5 \mathrm{~d}$ show the flux densities that result from multiplying the flux measurements shown in Fig. $4 \mathrm{a}$ and Fig. 5a by the window functions in Fig. $4 \mathrm{c}$ and Fig. $5 \mathrm{c}\left(W_{m a}, W_{m b}\right.$ and $W_{m c}$ ), (3)-(5). Fig. 4e and Fig. 5e show the zero-sequence component of flux density, $B_{m d q 0}(6)$, resulting from the flux densities shown in Fig. 4 d and Fig. 5 d.

$B_{m a}^{\prime}=B_{m a} W_{m a}$ 


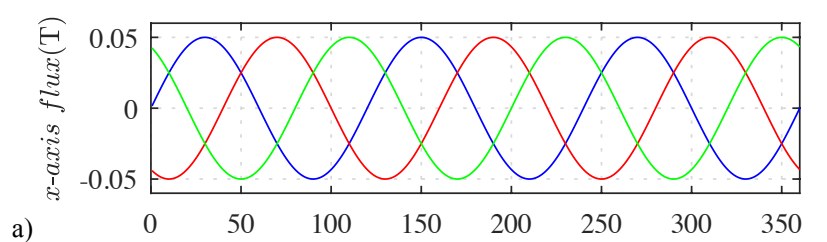

a)

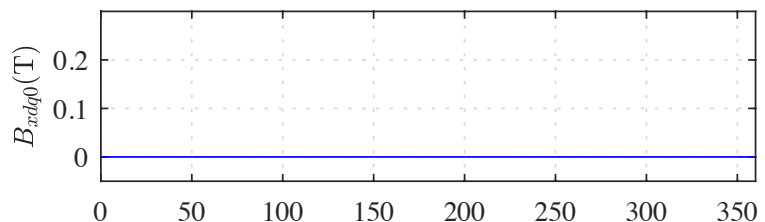

b)
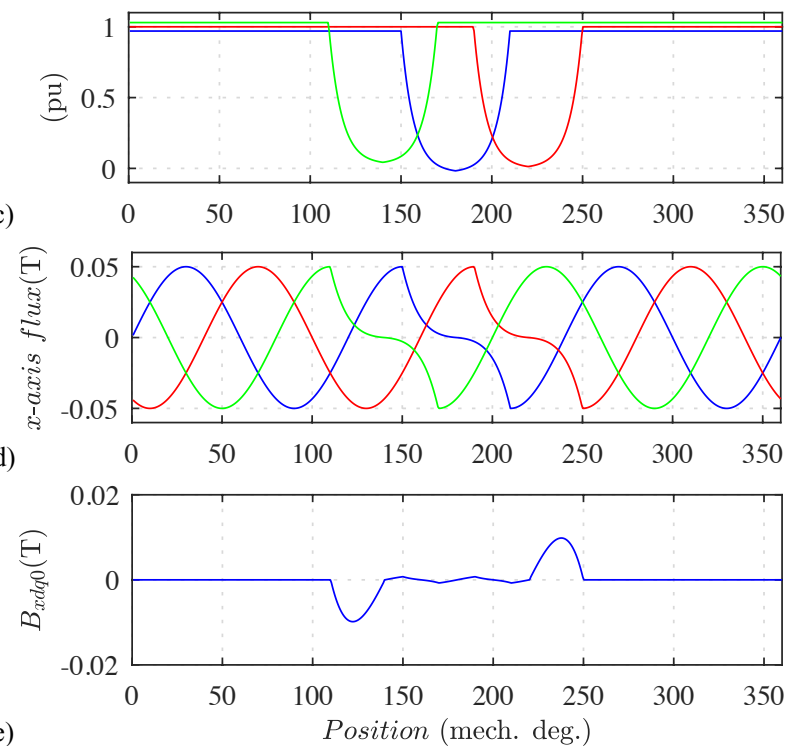

e)

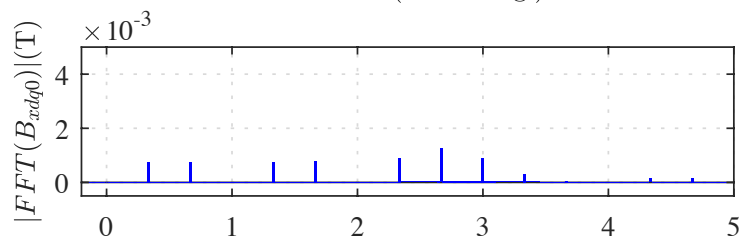

f)

$$
\text { Harmonic order }
$$

Fig. 4.- a) Ideal flux measurements in the $x$-axis direction by the three hall-effect sensors ( $B_{x a}, B_{x b}$ and $B_{x c}$ ) in a healthy machine, b) zerosequence magnetic flux density, $B_{x d q 0}$, resulting from the waveforms shown in a), c) profile of flux variation due to demagnetization (1 PM demagnetized by $50 \%$ ), d) flux in the $x$-axis direction by all the three analog hall-effect sensors for the case when 1 PM is demagnetized (i.e. multiplication of the flux measurements in a) by the window functions in c)), e) and f) magnitude and FFT of $B_{x d q 0}$ resulting from the waveforms shown in d). $\omega_{r}=1 \mathrm{pu}$.

$$
\begin{aligned}
B_{m b}^{\prime}= & B_{m b} W_{m b} \\
B_{m c}^{\prime}= & B_{m c} W_{m c} \\
B_{m d q 0} & =1 / 3\left(B_{m a} W_{m a}+B_{m a} W_{m a}+B_{m a} W_{m a}\right) \\
& =1 / 3\left(B_{m a}^{\prime}+a B_{m b}^{\prime}+B_{m c}^{\prime}\right)
\end{aligned}
$$

Different metrics can be defined, either in the time domain or in the frequency domain, from the processing of the zero sequence flux to assess PM condition. Simple metrics in the time domain include the mean value and peak-to-peak value

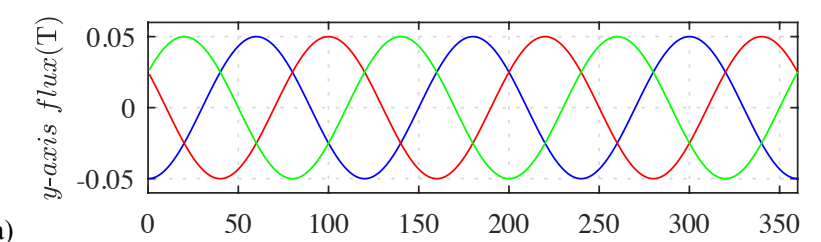

a)

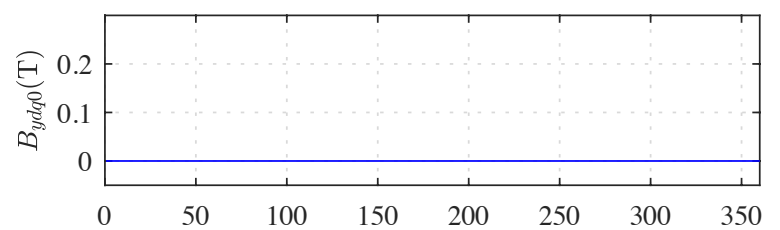

b)

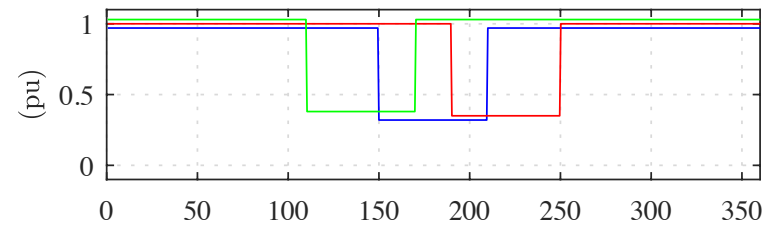

c)

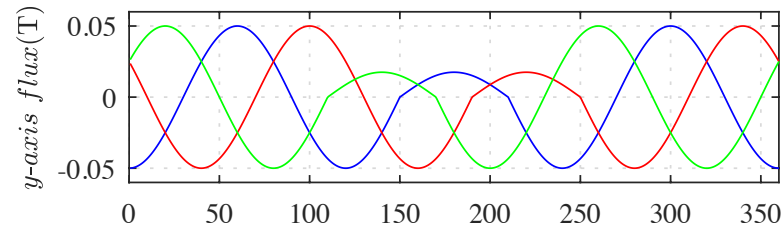

d)

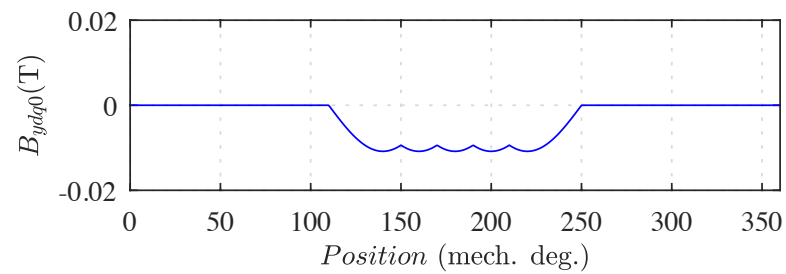

e)

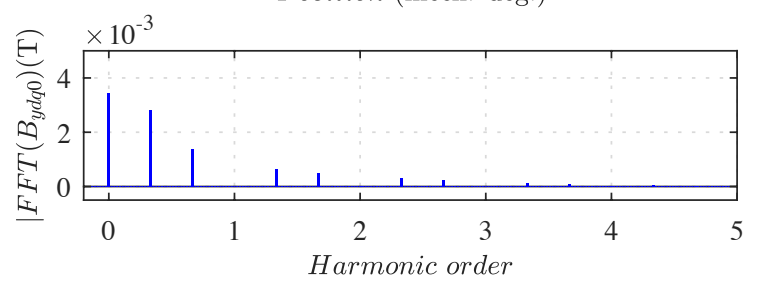

Fig. 5.- Same results as in Fig. 4 for the $y$-axis direction.

of the zero sequence flux (see Fig. 4e and 5e). In case of performing the analysis in the frequency domain, it is observed from Fig. $4 \mathrm{f}$ and Fig. $5 \mathrm{f}$ that demagnetization of PMs induces additional harmonic components in the spectrum of $B_{m d q 0}$. Tracking of such harmonics can be used to assess PM condition.

\section{Experimental verification of non-uniform demagnetization detection using the zero-sequence magnetic flux density}

Fig. 6 and Fig. 7 show experimental results when one of the PMs of the test machine (PM \#4 in Fig. 1) is demagnetized by $50 \%$ when the flux is measured in the $x$ and $y$-axis directions. It can be observed that the measurements in Fig. 6a and Fig. 7a are in good agreement with the patterns shown in Fig. $4 \mathrm{~d}$ and Fig. 5d. Fig. 6c and Fig. 7c show the FFTs of the zero-sequence 


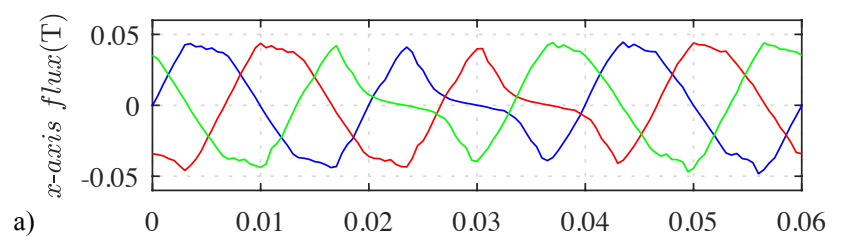

a)

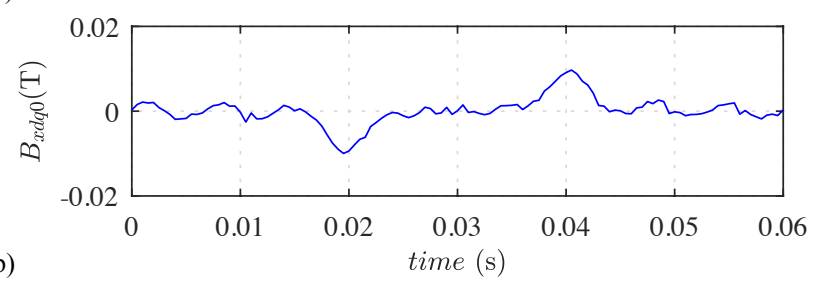

b)

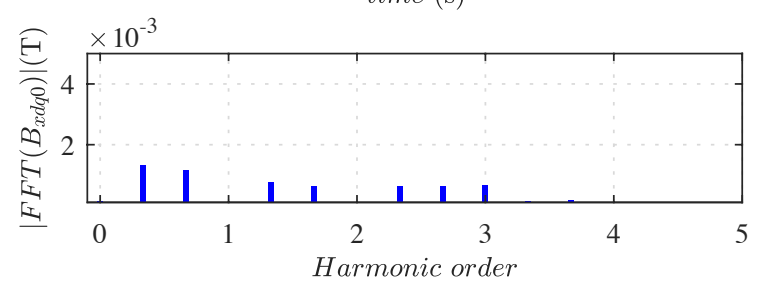

c)

Fig. 6.- a) flux measurements in the $x$-axis direction by the three analog hall effect sensors ( $B_{x a}, B_{x b}$ and $\left.B_{x c}\right)$, b) resulting zero-sequence magnetic flux density, $B_{x d q 0}$, and c) FFT of $B_{x d q 0}$. Sensor location: $x=0 \mathrm{~mm}, y=0 \mathrm{~mm}, z=5 \mathrm{~mm} ;$ machine operating condition: $\omega_{r}=1 \mathrm{pu}$ and $i_{d q}=0 \mathrm{pu}, 1 \mathrm{PM}$ demagnetized by $50 \%$.
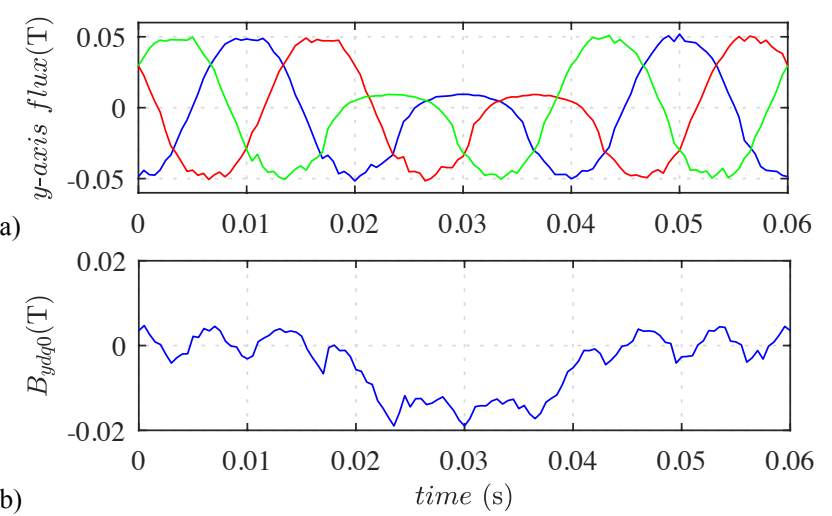

b)

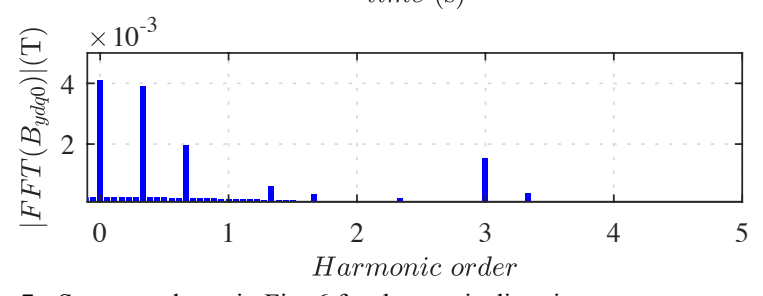

Fig. 7.- Same results as in Fig. 6 for the $y$-axis direction.

flux, which are also seen to be in good agreement with the predictions shown in Fig. $4 \mathrm{f}$ and Fig. $5 f$.

Fig. $8 \mathrm{a}$ and Fig. $8 \mathrm{~b}$ show the variation of different metrics using the flux zero sequence when the demagnetization of PM \#4 goes from zero (fully magnetized PM) to $50 \%$. Fig. $8 \mathrm{a}$ shows the mean value of $B_{m d q 0}$ and $\left|B_{m d q 0}\right|$ as demagnetization degree of PM \#4 increases. As expected, the mean value of $B_{x d q 0}$ is not useful as it is a symmetric waveform (see Fig. 6a). On the other hand, mean values of $\left|B_{x d q 0}\right|, B_{y d q 0}$ and $\left|B_{y d q 0}\right|$ change almost linearly with the demagnetization state, allowing reliable demagnetization detection even for low

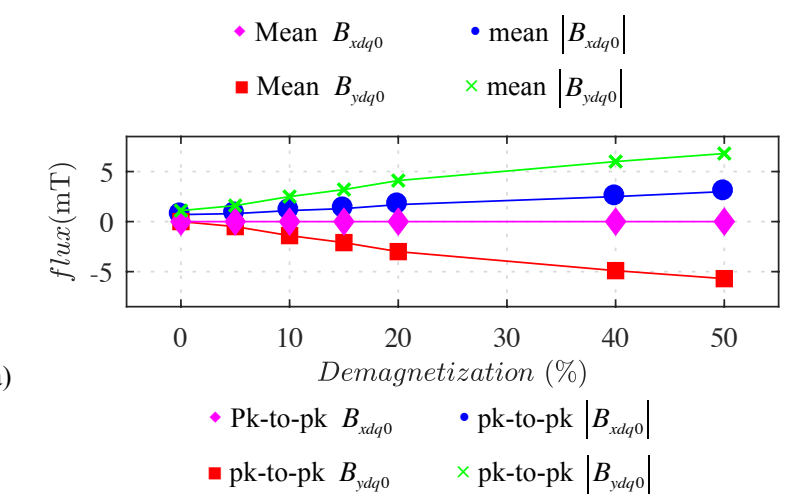

b)

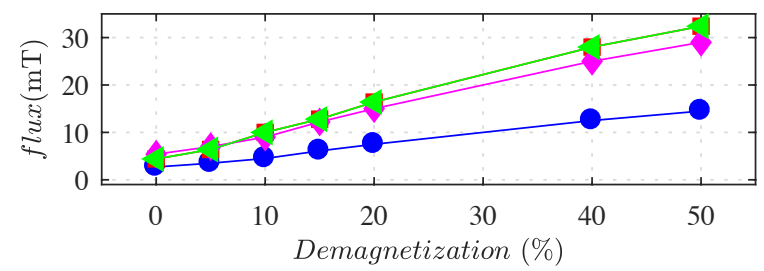

Fig. 8.- a) mean and b) peak-to-peak of the zero-sequence magnetic flux density, $B_{m d q 0}$, and absolute value of the zero-sequence magnetic flux density, $\left|B_{m d q 0}\right|$, vs. PM \#4 demagnetization level. Sensor location: $x=0 \mathrm{~mm}, y=0 \mathrm{~mm}, z=5 \mathrm{~mm}$; machine operating condition: $\omega_{r}=1 \mathrm{pu}$ and $i_{d q}=0 \mathrm{pu}$.

demagnetization levels. Fig. $8 \mathrm{~b}$ shows the peak-to-peak of $B_{m d q 0}$ and $\left|B_{m d q 0}\right|$ both signals being also almost a linear function of the demagnetization degree.

Fig. 9 shows the variation of the same metrics shown in Fig. 8 but when the number of demagnetized PMs increase: PMs are demagnetized sequentially from PM \#1 to \#6, see Fig. 1; magnetization state of demagnetized PMs being set to $80 \%$. As expected from Fig. $8 \mathrm{a}$, the mean value of $B_{x d q 0}$ is not useful for demagnetization detection. It is observed that the mean value of $B_{y d q 0}$ is higher when the number of demagnetized PMs is odd than when it is even; this is explained because when the number of demagnetized PMs is even, a complete pole pair is demagnetized, $B_{y d q 0}$ waveform being therefore symmetric, as expected from Fig. 5. It can be therefore concluded that mean value of $B_{y d q 0}$ is a reliable metric to distinguish faults which affect to a complete pole or pole pair.

Mean values of $\left|B_{x d q 0}\right|$ and $\left|B_{y d q 0}\right|$ are almost zero when there are no demagnetized PMs or when the demagnetization is uniform, i.e. uniform demagnetization cannot be distinguished using this metric. In addition, both are seen to be almost independent of the number of demagnetized magnets. It is concluded that these are reliable metrics of the demagnetization degree (see Fig. 8a), but fail to show the number of PMs that have been demagnetized.

It is observed from Fig. $9 \mathrm{~b}$ that the peak-to-peak value of $B_{m d q 0}$ and $\left|B_{m d q 0}\right|$ shows a relative small value when the number of demagnetized PMs is 0 or 6 , i.e. uniform demagnetization cannot be distinguished using this metric. It is also observed from Fig. $9 \mathrm{~b}$ that the peak-to-peak of $B_{m d q 0}$ and $\left|B_{m d q 0}\right|$ show almost no dependence on the number of demagnetized magnets. Consequently, they can be a reliable 

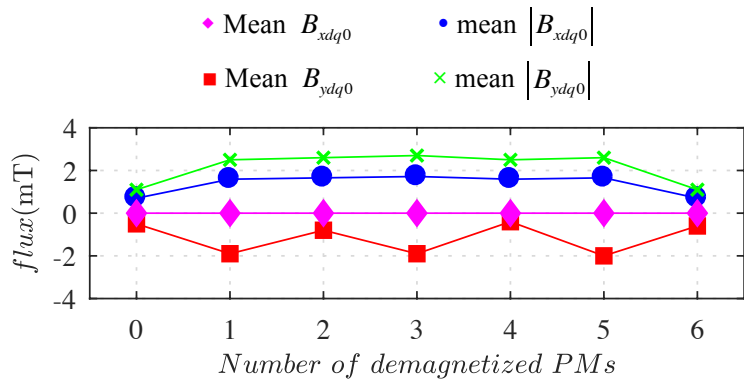

a)

$$
\begin{array}{ll}
\text { - Pk-to-pk } B_{x d q 0} & \bullet \text { pk-to-pk }\left|B_{x d q 0}\right| \\
\text { - pk-to-pk } B_{y d q 0} & \times \text { pk-to-pk }\left|B_{y d q 0}\right|
\end{array}
$$

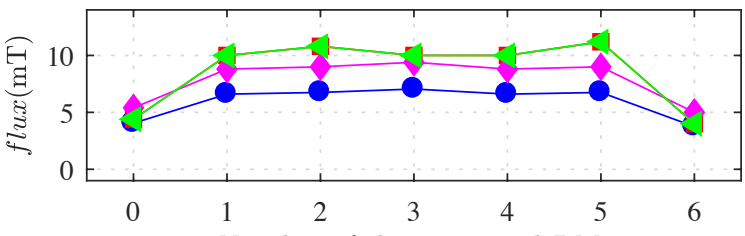

b)

Number of demagnetized PMs

Fig. 9.- a) mean and b) peak-to-peak of the zero-sequence magnetic flux density, $B_{m d q 0}$, and absolute value of the zero-sequence magnetic flux density, $\left|B_{m d q 0}\right|$, vs. number of demagnetized PMs (magnetization level $=90 \%$ ). Sensor location: $x=0 \mathrm{~mm}, \quad y=0 \mathrm{~mm}, z=5 \mathrm{~mm}$; machine operating condition: $\omega_{r}=1 \mathrm{pu}$ and $i_{d q}=0 \mathrm{pu}$.

metric for the demagnetization degree (see Fig. 8b), but not of the number of PMs demagnetized.

Table II summarizes the properties of the different metrics.

\begin{tabular}{|c|c|c|c|c|c|}
\hline \multicolumn{6}{|c|}{ Table II. Metrics to detect demagnetization } \\
\hline & $\begin{array}{c}\text { Uniform } \\
\text { demag. }\end{array}$ & $\begin{array}{l}\text { Non- } \\
\text { uniform } \\
\text { demag. }\end{array}$ & $\begin{array}{c}\text { Asymmetries } \\
\text { among } \\
\text { poles }\end{array}$ & $\begin{array}{c}\text { Asymmetries } \\
\text { among } \\
\text { pole pairs }\end{array}$ & $\begin{array}{l}\text { Demag. } \\
\text { degree }\end{array}$ \\
\hline Mean $B_{x d q 0}$ & $x$ & $x$ & $x$ & $x$ & $x$ \\
\hline Mean $\left|B_{x d q 0}\right|$ & $x$ & $\checkmark$ & $x$ & $x$ & $\checkmark$ \\
\hline Mean $B_{y d q 0}$ & $x$ & $\checkmark$ & $\checkmark$ & $x$ & $\checkmark$ \\
\hline mean $\left|B_{y d q 0}\right|$ & $x$ & $\checkmark$ & $x$ & $x$ & 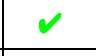 \\
\hline Pk-to-pk $B_{x d q 0}$ & $x$ & $\checkmark$ & $x$ & $x$ & $\checkmark$ \\
\hline Pk-to-pk $\left|B_{x d q 0}\right|$ & $x$ & $\checkmark$ & $x$ & $x$ & $\checkmark$ \\
\hline Pk-to-pk $B_{y d q 0}$ & $x$ & $\checkmark$ & $x$ & $x$ & $\checkmark$ \\
\hline Pk-to-pk $\left|B_{y d q 0}\right|$ & $x$ & $\checkmark$ & $x$ & $x$ & $\checkmark$ \\
\hline
\end{tabular}
Refinement of these metrics is a subject of ongoing research.

\section{Conclusions}

Demagnetization detection based on the zero-sequence magnetic flux measurement has been analyzed in this work. The method uses standard hall-effect sensors used in PMSMs drives for motion control have been used, and can be implemented therefore without additional hardware. Extensive experimental results have been provided to validate the proposed method. It has been shown that simple metrics as the peak-to-peak value or the mean value of the zerosequence magnetic flux density can provide reliable estimations of the magnetization state.

\section{References}

[1] J. F. Gieras and M. Wing, "Permanent magnet motor technology: design and application". Second edition 2002.

[2] D. W. Novotny and T. A. Lipo, "Vector Control and Dynamics of AC Drives," Oxford Science Publications, 1996.

[3] K. Akatsu, M. Arimitsu, and S. Wakui, "Design and control of a field intensified interior permanent magnet synchronous machine," IEEJ Trans. Ind. Appl., 126(7): 827-834, Jul. 2006.

[4] D. Reigosa, F. Briz, M. W. Degner, P. García and J. M. Guerrero, "Magnet temperature estimation in surface PM machines during sixStep operation". IEEE Trans. on Ind. Appl., 48(6): 2353-2361, Nov.Dec. 2012.

[5] D. Reigosa, F. Briz, M. W. Degner, P. García and J. M. Guerrero, "Temperature issues in saliency-tracking-based sensorless methods for PM synchronous machines". IEEE Trans. on Ind. Appl., 47(3): 13521360, May-June 2011.

[6] D. Reigosa, D. Fernandez, H. Yoshida, T. Kato and F. Briz "PermanentMagnet Temperature Estimation in PMSMs Using Pulsating HighFrequency Current Injection,” IEEE Trans. on Ind. Appl., 51(4): 31593168, July-Aug 2015.

[7] N. Limsuwan, T. Kato, K. Akatsu, and R. D. Lorenz, "Design and evaluation of a variable-flux flux-intensifying interior permanentmagnet machine," IEEE Trans. Ind. Appl., 50(2): 1015-1024, Mar./Apr. 2014.

[8] Athavale, K. Sasaki, B. S. Gagas, T. Kato, and R. D. Lorenz, "Variable Flux Permanent Magnet Synchronous Machine (VF-PMSM) Design Methodologies to Meet Electric Vehicle Traction Requirements with Reduced Losses," IEEE Trans. Ind. Appl., 53(5): 4318-4326, Sep./Oct. 2016.

[9] S. Ruoho, J. Kolehmainen, J. Ikaheimo and A. Arkkio,"Interdependence of Demagnetization, Loading, and Temperature Rise in a PermanentMagnet Synchronous Motor," IEEE Trans. Magn., vol. 46, no. 3, pp. 949-953, Mar. 2010.

[10] J Hong, D Hyun, and S.B.Lee, "Automated monitoring of magnet synchronous motors at standstill," IEEE Trans. Ind. Appl., 46 (4): 1397 1405,2010

[11] IEC 62. 2-2004., "IEEE guide for diagnostic field testing of electric power apparatus-electrical machinery," 2004

[12] D. Fernandez, D. Reigosa, T. Tanimoto, T. Kato, and F. Briz, "Wireless permanent magnet temperature \& field distribution measurement system for IPMSMs," IEEE ECCE, pp. 3996-4003, Sep. 2015.

[13] X. Xiao, C. Chen and M. Zhang, "Dynamic Permanent Magnet Flux Estimation of Permanent Magnet Synchronous Machines", IEEE Trans. on Appl. Sup., 20(3): 1085-1088, June. 2010.

[14] K. Liu and Z. Q. Zhu "Online Estimation of the Rotor Flux Linkage and Voltage-Source Inverter Nonlinearity in Permanent Magnet Synchronous Machine Drives," IEEE Trans. on Pow. Elect., 29(1): 418427, Jan. 2014.

[15] K. Liu, Q. Zhang, J. Chen, Z. Q. Zhu, and J. Zhang, "Online multiparameter estimation of nonsalient-pole PM synchronous machines with temperature variation," IEEE Trans. Ind. Electron., vol. 58, no. 5, pp. 1776-1788, May 2011.

[16] D. Reigosa, D. Fernandez, J. M. Guerrero, Z.Q. Zhu and F. Briz, "PMSM Magnetization State Estimation Based on Stator-reflected PM Resistance Using High Frequency Signal Injection", IEEE Trans. on Ind. Appl., 51(5): 3800-3810, Sept.-Oct. 2015.

[17] D. Fernandez, D. Reigosa, Z.Q. Zhu and F. Briz, "Permanent-Magnet Magnetization State Estimation Using High-Frequency Signal Injection”, IEEE Trans. on Ind. Appl., 52(4): 2930-2949, July-Aug. 2016.

[18] D. Reigosa, D. Fernandez, Yonghyun Park, A. B. Diez, S. B. Lee and F. Briz, "Detection of Demagnetization in Permanent Magnet Synchronous Machines using Hall-Effect Sensors" IEEE Trans. on Ind. Appl., 54(4): Early Access, 2018. DOI: 10.1109/ECCE.2017.8096799.

[19] J.-R. R. Ruiz, A. G. Espinosa, L. Romeral, and J. Cusidó, "Demagnetization diagnosis in permanent magnet synchronous motors under nonstationary speed conditions," Electr. Power Syst. Res., 80(10): 12771285, Oct. 2010.

[20] S. Rajagopalan, W. le Roux, T. G. Habetler, and R. G. Harley, "Dynamic eccentricity and demagnetized rotor magnet detection in trapezoidal flux (brushless DC) motors operating under different load conditions,” IEEE Trans. Power Electron., 22(5): 2061-2069, Sep. 2007. 
[21] C. Urresty, J.-R. R. Ruiz, M. Delgado, and L. Romeral, "Detection of demagnetization faults in surface-mounted permanent magnet synchronous motors by means of the zero-sequence voltage component," IEEE Trans. Energy Convers., 27(1): 42-51, Mar. 2012.

[22] J. C. Urresty, J.-R. R. Ruiz, and L. Romeral, "A back-EMF based method to detect magnet failures in PMSMs," IEEE Trans. Magn., 49(1): 591-598, Jan. 2013.

[23] Z. Yang, X. Shi, and M. Krishnamurthy, "Vibration monitoring of PM synchronous machine with partial demagnetization and inter-turn short circuit faults," in Proc. IEEE ITEC, pp. 1-6, June 2014.

[24] D. Torregrossa, A. Khoobroo and B Fahimi, "Prediction of Acoustic Noise and Torque Pulsation in PM Synchronous Machines With Static Eccentricity and Partial Demagnetization Using Field Reconstruction Method”, IEEE Trans. on Ind. Elect., 59(2): 934-944, Feb. 2012.

[25] J. C. Urresty, R. Atashkhooei, J.-R. R. Ruiz, L. Romeral, and S. Royo, "Shaft trajectory analysis in a partially demagnetized permanent-magnet synchronous motor," IEEE Trans. Ind. Electron., 60(8): 3454-3461, Aug. 2013.

[26] J. Hong, D. Hyun, S.B. Lee, J.Y. Yoo and K.W. Lee, "Automated Monitoring of Magnet Quality for Permanent-Magnet Synchronous Motors at Standstill," IEEE Trans. Ind. Appl., 46(4): 1397-1405, JulyAug. 2010.

[27] Maxonmotorusa.com, "maxon sensor - Key information," Mar. 23, 2017. [Online]. Available: http://www.maxonmotorusa.com/

[28] Siemens.com, "Three-phase synchronous motors based on permanent magnet technology," Catalog D 86.2 • 2007, Mar. 23, 2017. [Online]. Available: http://w3.siemens.com/mcms/mc-solutions/en/motors/

[29] L. Xiao, Y. Yunyue, Z. Zhuo, "Study of the Linear Hall-Effect Sensors Mounting Position for PMLSM," IEEE Conference on Industrial Electronics and Applications, pp. 1175 - 1178, May 2017.

[30] http://www.allegromicro.com/en/Products/Magnetic-Linear-AndAngular-Position-Sensor-ICs/Linear-Position-Sensor-ICs/A1301-2.aspx 